\title{
Comparative clinical profile of mirtazapine and duloxetine in practical clinical settings in Japan: a 4-week open-label, parallel-group study of major depressive disorder
}

\author{
This article was published in the following Dove Press journal: \\ Neuropsychiatric Disease and Treatment \\ 4 June 2013 \\ Number of times this article has been viewed
}

\author{
Kei Nagao ${ }^{1,2}$ \\ Taro Kishi' \\ Masatsugu Moriwaki' \\ Kiyoshi Fujita ${ }^{3,4}$ \\ Shigeki Hirano 5 \\ Yoshio Yamanouchi' \\ Toshihiko Funahashi² \\ Nakao Iwatal \\ 'Department of Psychiatry, Fujita \\ Health University School of Medicine, \\ Toyoake, Aichi, Japan; '2Department \\ of Psychiatry, The Jindai Hospital, \\ Toyota, Aichi, Japan; ${ }^{3}$ Department \\ of Psychiatry, The Okehazama \\ Hospital, Toyoake, Aichi, Japan; \\ ${ }^{4}$ The Neuroscience Research Center, \\ Toyoake, Aichi, Japan; ${ }^{5}$ Department \\ of Psychiatry, The Toyota Memorial \\ Hospital, Toyota, Aichi, Japan
}

\begin{abstract}
No studies have compared mirtazapine with duloxetine in patients with major depressive disorder (MDD). Fifty-six patients were nonrandomly assigned to a 4-week treatment with either 15 to $45 \mathrm{mg} /$ day of mirtazapine $(\mathrm{n}=22)$ or 20 to $60 \mathrm{mg} /$ day of duloxetine $(n=34)$. The primary efficacy measurements were the Hamilton Rating Scale for Depression (HRSD) and the Montgomery-Åsberg Depression 6-point Rating Scale (MADRS) scores. The second efficacy measurements were the response and remission rates of treatment. Tolerability assessments were also performed. Fifty-six patients (43 male; age, 43.6 years) were recruited. There was no significant difference in the discontinuation rate between the mirtazapine and duloxetine treatment groups $(P=0.867)$. Both mirtazapine and duloxetine significantly improved the HRSD and MADRS scores from baseline $(P<0.0001-0.0004)$. While mirtazapine was superior to duloxetine in the reduction of HRSD scores $(P=0.0421)$, there was no significant change in MADRS scores in terms of between-group differences $(P=0.171)$. While more somnolence was observed with mirtazapine $(P=0.0399)$, more nausea was associated with duloxetine $(P=0.0089)$. No serious adverse events were observed for either antidepressant. Mirtazapine and duloxetine were safe and well-tolerated treatments for Japanese patients with MDD. Double-blind controlled studies are needed to further explore the efficacy and safety of mirtazapine and duloxetine in Japanese patients with MDD.
\end{abstract}

Keywords: mirtazapine, duloxetine, major depressive disorder

\section{Introduction}

In July 2009 and April 2010, mirtazapine and duloxetine, respectively, were approved as antidepressants in Japan for the management of major depressive disorder (MDD). Mirtazapine is classified as a noradrenergic and specific serotonergic antidepressant (NaSSA). NaSSAs act by antagonizing various adrenergic and serotonin receptors, which typically comprise $\alpha 1$-adrenergic and $\alpha 2$-adrenergic receptors and serotonin $(5-\mathrm{HT})_{2 \mathrm{~A}}, 5-\mathrm{HT}_{2 \mathrm{C}}$, and $5-\mathrm{HT}_{3}$ receptors, respectively. By blocking $\alpha 2$-adrenergic autoreceptors and heteroreceptors, NaSSAs enhance adrenergic and serotonergic neurotransmission, notably $5-\mathrm{HT}_{1 \mathrm{~A}}$-mediated transmission. ${ }^{1}$ Unlike many common antidepressants, NaSSAs have no efficacy as serotonin-reuptake inhibitors. According to a recent meta-analysis, while mirtazapine did not show a greater efficacious response rate or remission rate compared with tricyclic antidepressants (TCAs), mirtazapine was inferior to selective serotonin-reuptake inhibitors (SSRI) in terms of their response rate. ${ }^{2}$ On the other hand, duloxetine is a well-known selective serotonin and noradrenaline-reuptake inhibitor (SNRI). ${ }^{3}$ According to
Correspondence: Taro Kishi Department of Psychiatry, Fujita Health University School of Medicine, Toyoake, Aichi 470-I 192, Japan

Tel +8I 562939250

Fax +8| $56293|83|$

Email tarok@fujita-hu.ac.jp 
another recent meta-analysis, there was no significant difference in the efficacy or safety between duloxetine and SSRIs. ${ }^{3}$ Conversely, the Meta-Analyses of New Generation Antidepressants (MANGA) study (a multiple treatment meta-analysis and statistical technique that allows for both direct and indirect comparisons even when two of the treatments have not been directly compared) reported that mirtazapine was significantly more efficacious than duloxetine. ${ }^{4}$ However, there was no direct comparison between mirtazapine and duloxetine. The antidepressant effect of both drugs is considered to be due to increased serotonin and noradrenaline in the synaptic cleft. However, as stated above, the pharmacological action of both drugs is different. Therefore, we conducted a 4-week open-label, parallel-group clinical study involving direct comparisons of mirtazapine and duloxetine focusing on the clinical profile of the onset of action and tolerability in patients with MDD in routine practical clinical settings in Japan.

\section{Method}

\section{Subjects}

The present study was conducted from November 2010 to May 2012. Patients were recruited from a cohort of patients in Fujita Health University, The Jindai Hospital, The Okehazama Hospital, and The Toyota Memorial Hospital in Japan. The patients were diagnosed according to the Diagnostic and Statistical Manual of Mental Disorders (fourth edition) criteria for MDD with the consensus of at least two experienced psychiatrists on the basis of an unstructured interview and a review of all medical records. Patients with bipolar disorder were excluded. All subjects met the following inclusion criteria: (1) a score of $>15^{5}$ on the Hamilton Rating Scale for Depression (HRSD) 5-point scale, ${ }^{6}(2)$ age of 20 to 70 years, (4) no systemic or neurologic diseases, including disturbances of hematopoiesis, (5) no history of electroconvulsive therapy within 6 months prior to study enrollment, (6) not pregnant, and (7) no dependence on any substances other than nicotine during the 5 years before enrollment. No patient was excluded from the study because of a medical condition at baseline.

Each clinician explained the study purpose and design to patients eligible for this study, and informed consent was obtained from each patient before participating in the study. The trial was conducted in accordance with good clinical practices and the Helsinki Declaration. This study was approved by the ethics committees at Fujita Health University and the Jindai, Toyota Memorial, and Okehazama hospitals.

\section{Procedures and evaluation of psychopathology, tolerability, and safety}

This study was open-label and active-controlled. Patients were nonrandomly assigned to one of two treatment groups. Mirtazapine or duloxetine was taken once a day for 4 weeks. Mirtazapine and duloxetine were initiated at daily doses of 15 (at bedtime) and $20 \mathrm{mg} /$ day (after breakfast), respectively. After 1 week, the dose was flexibly altered by each doctor based on the patient's systemic clinical conditions and side effects at each visit (weeks 1, 2, and 4). The doses of mirtazapine and duloxetine were gradually increased to maximums of 45 and $60 \mathrm{mg}$, respectively, depending on the patient's condition. The authorized usual daily doses of mirtazapine and duloxetine for patients with MDD in Japan are 15 to $45 \mathrm{mg}$ and 20 to $60 \mathrm{mg}$, respectively. Therefore, these doses were used for this study. Patients with severe insomnia were prescribed zolpidem or brotizolam, but no other psychotropic drugs were permitted during the study. Zolpidem and brotizolam were used within the Japanese authorized dose ranges, ie, 5 to $10 \mathrm{mg}$ for zolpidem and $0.25 \mathrm{mg}$ for brotizolam at bedtime.

Patients were assessed for depressive symptoms with the HRSD and the Montgomery-Åsberg Depression 6-point Rating Scale (MADRS) ${ }^{7}$ as primary efficacy measurements at baseline and at 1,2, and 4 weeks. The second efficacy measurements were the treatment response rate and the remission rate. A clinical response was defined as a decrease of $>50 \%$ in the baseline HRSD score within 4 weeks, ${ }^{8}$ and clinical remission was defined as an HRSD score of $<7$ at 4 weeks. ${ }^{8,9}$ Patients' systemic subjective clinical conditions and side effects were monitored by full physical examination including vital signs and general questioning regarding adverse events at each visit (weeks 1,2, and 4). The clinical characteristics of the patients in this study, classified according to these definitions, are shown in Table 1.

\section{Statistical analysis}

An intent-to-treat analysis was performed using the lastobservation-carried-forward method. Subjects with at least two measurement points were entered into the study analyses. Each participant's last observation was taken as the endpoint. The percentage change in the HRSD and MADRS scores from baseline to endpoint was not significantly different from a normal distribution according to the Shapiro-Wilk test. The paired Student's $t$ test was used to assess the significance of the change in psychopathology scores from baseline to endpoint in each treatment group. To determine 
Table I Participant characteristics at baseline

\begin{tabular}{|c|c|c|c|}
\hline & Mirtazapine & Duloxetine & $P$ \\
\hline$N$ & 22 & 34 & \\
\hline Male $(\% \text { male })^{b}$ & I8 (8।.8) & $25(73.5)$ & 0.535 \\
\hline Age (years, mean $\pm S D)^{a}$ & $43.6 \pm 13.0$ & $43.6 \pm 14.3$ & 0.999 \\
\hline $\begin{array}{l}\text { Age at onset } \\
(\text { years, mean } \pm S D)^{\text {a }}\end{array}$ & $41.1 \pm 10.3$ & $42.7 \pm 14.2$ & 0.650 \\
\hline 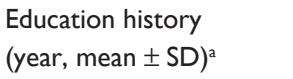 & $13.7 \pm 2.3$ & $14.1 \pm 2.1$ & 0.505 \\
\hline $\begin{array}{l}\text { Initial dose } \\
\text { (mg/day, mean } \pm S D)\end{array}$ & $15 \pm 0.00$ & $20 \pm 0.00$ & na \\
\hline Alcohol dependence $^{b}$ & 0 & 0 & na \\
\hline $\begin{array}{l}\text { Hospitalization } \\
\text { at baseline (\%) }\end{array}$ & $5(22.7)$ & I (2.9) & 0.0299 \\
\hline $\begin{array}{l}\text { Number of episodes } \\
(\text { mean } \pm S D)^{\mathrm{a}}\end{array}$ & $0.455 \pm 0.702$ & $0.182 \pm 0.458$ & 0.0833 \\
\hline $\begin{array}{l}\text { Employment rate at } \\
\text { baseline (\%) }\end{array}$ & $12(75.0)$ & $21(75.0)$ & 0.722 \\
\hline $\mathrm{HRSD}^{\mathrm{a}}$ & $25.1 \pm 6.84$ & $20.4 \pm 5.62$ & 0.0069 \\
\hline MADRS $^{\mathrm{a}}$ & $36.5 \pm 8.64$ & $28.1 \pm 8.04$ & 0.0005 \\
\hline
\end{tabular}

Notes: 'at-test; 'Fisher's exact test.

Abbreviations: HRSD, Hamilton Rating Scale for Depression; MADRS, Montgomery-Åsberg Depression Rating Scale; na, not applicable; SD, standard deviation.

which antidepressant was more effective for the psychopathology of the patients with MDD, we used linear regression to analyze the relationship between each antidepressant and changes in the final HRSD and/or MADRS total scores. For both mirtazapine and duloxetine, significant differences with HRSD or MADRS scores at baseline were detected (Table 1). Analyses for comparison between the treatment groups were conducted using the mixed-model repeated measures (MMRM) approach. For the MMRM analyses, changes from baseline at all postbaseline times were the dependent variables. Independent variables included treatment group, week, HRSD or MADRAS score at baseline, age at recruitment, sex, and the treatment-group-by-week interaction. The error matrix was unstructured. The analyses of the response and remission rates were performed using Fisher's exact test. All statistical tests were carried out via JMP (JMP 5.0. $1 \mathrm{~J}$; SAS Japan Inc, Tokyo, Japan), and all $P$ values of $<0.05$ were considered statistically significant.

\section{Results}

Patient demographics and distributions are presented in Table 1. Fifty-six patients (43 male; age, 43.6 years) were recruited for inclusion in the trial. There were significant differences in the HRSD and MADRS scores at baseline and the number of hospitalizations at baseline between the two treatment groups (Table 1). Six of 23 patients (26.1\%) in the mirtazapine group and nine of 33 patients $(28.1 \%)$ in the duloxetine group were dropped from the study because of any cause (Figure 1). One of 22 patients $(4.6 \%)$ in the mirtazapine group and three of 34 patients $(8.8 \%)$ in the duloxetine group were dropped from the study because of inefficacy. Four of 22 patients (18.2\%) in the mirtazapine group and four of 34 patients $(11.8 \%)$ in the duloxetine group were dropped from the study because of side effects. However, there were no significant differences in the discontinuation rate due to any cause $(P=0.867)$, inefficacy ( $P=0.485)$, and side effects $(P=0.662)$ between the groups. The mean mirtazapine and duloxetine doses at 4 weeks were

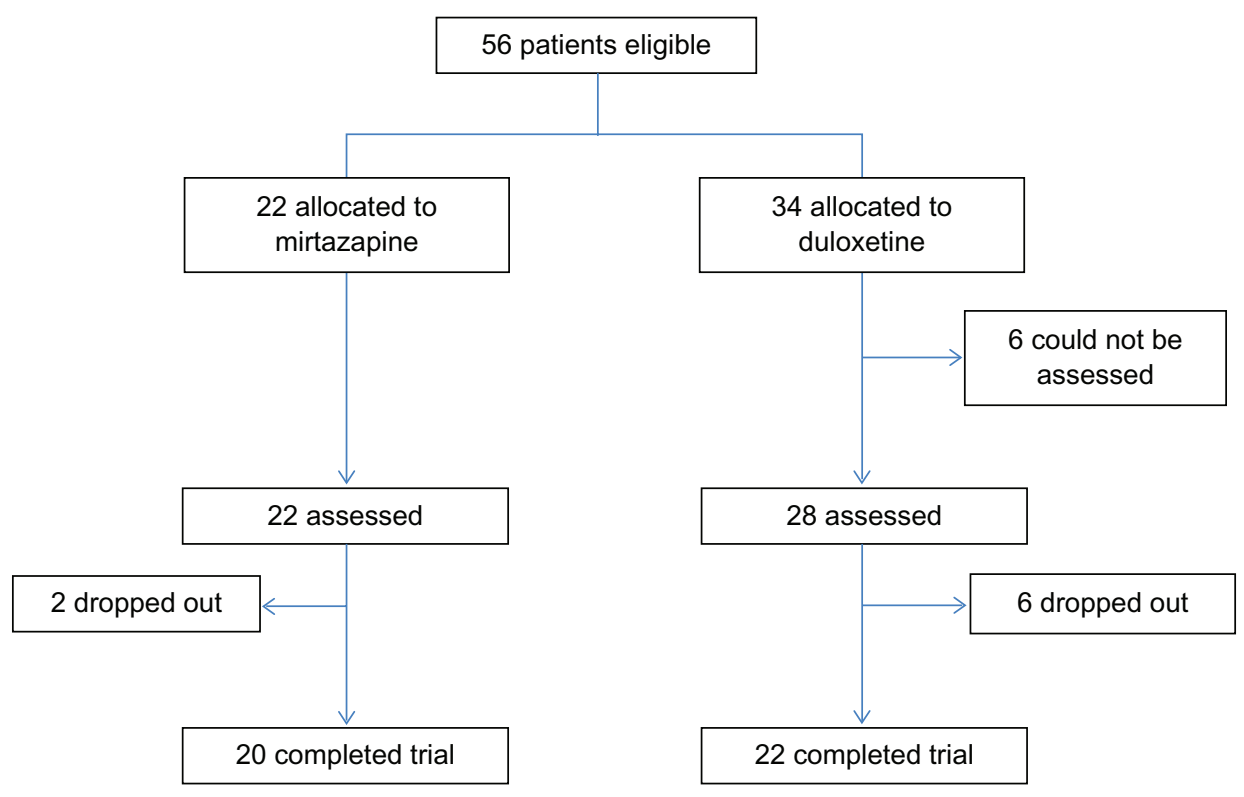

Figure I Patients disposition. 


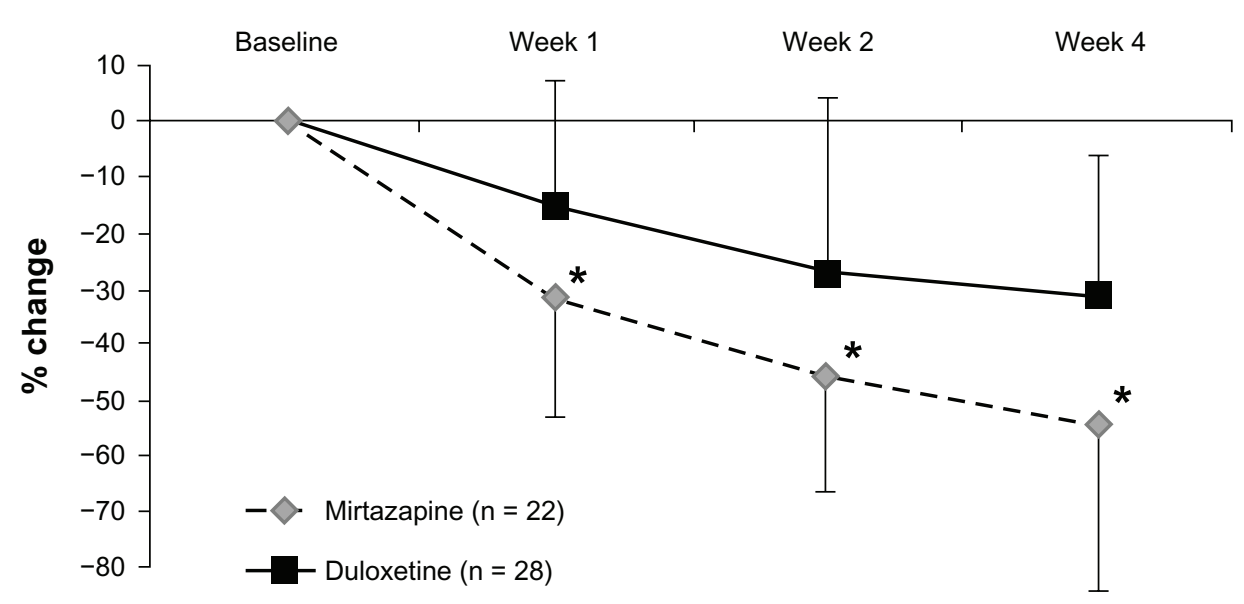

Figure 2 Change from baseline to weeks I, 2, and 4 in the total scores of the Hamilton Rating Scale for Depression.

Note: $* P<0.05$ (MMRM).

Abbreviation: MMRM, mixed-models repeated measures.

$27 \pm 10.4$ and $37.6 \pm 8.79 \mathrm{mg} /$ day, respectively. Eleven patients $(50.0 \%)$ in the mirtazapine group and 26 patients $(76.5 \%)$ in the duloxetine group were taking sleeping pills during the trial $(P=0.0495)$.

Both mirtazapine and duloxetine significantly improved the HRSD and MADRS scores from baseline. While mirtazapine was superior to duloxetine in the reduction of HRSD scores (Figure 2), there was no significant change in MADRS scores in terms of between-group differences (Figure 3, Table 2). In addition, there were no significant differences in the response rate (mirtazapine, $59.1 \%$; duloxetine, $28.6 \%$ ) or remission rate (mirtazapine, $31.8 \%$; duloxetine, 10.7\%) between the two groups (Table 2).

While more somnolence was observed with mirtazapine $(P=0.0399)$, more nausea was associated with duloxetine $(P=0.0089)$. We did not find any other differences in side effects, including insomnia, dry mouth, weight gain, and dizziness, between the treatment groups. No serious adverse events were observed for either medication.

\section{Discussion}

This is the first comparison between mirtazapine and duloxetine to focus on efficacy and safety in patients with MDD. Both antidepressants were beneficial and relatively well tolerated in patients with MDD in Japan. Unfortunately, there were significant differences in the HRSD and MADRS scores at baseline. Therefore, we controlled for these confounding factors by evaluating the change in HRSD and MADRS scores from baseline using MMRM. Although there was a difference in the number of patients who discontinued the trial between the two treatment groups, mirtazapine seemed to be more acceptable. While mirtazapine was superior to duloxetine in terms of the reduction in HRSD scores (Figure 2), there was no significant change in MADRS scores

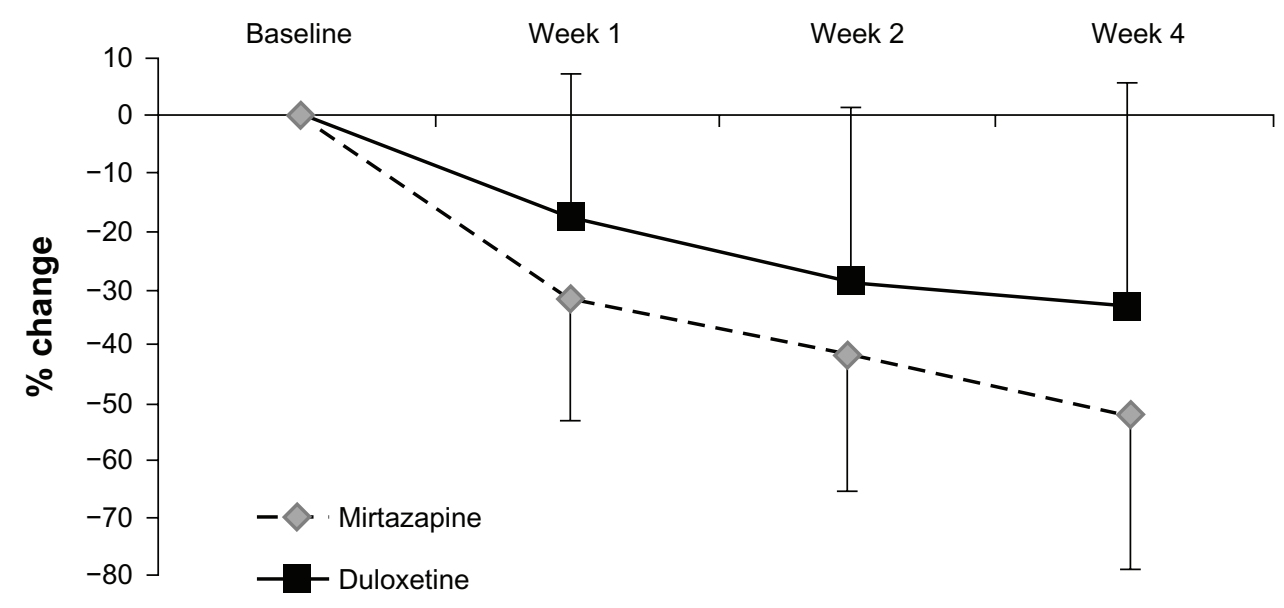

Figure 3 Change from baseline to weeks I, 2, and 4 in the total scores of the Montgomery-Åsberg Depression Rating Scale. 
Table 2 Efficacy: change from baseline to weeks I, 2, and 4 in the total scores of Hamilton Rating Scale for depression and MontgomeryÅsberg Depression Rating Scale, and response and remission rates

\begin{tabular}{|c|c|c|c|c|c|}
\hline & \multicolumn{2}{|l|}{ Mirtazapine } & \multicolumn{2}{|l|}{ Duloxetine } & \multirow{2}{*}{$\begin{array}{l}P \text { value for between-treatment } \\
\text { group comparisons regarding } \\
\text { change from baseline (MMRM) }\end{array}$} \\
\hline & Mean \pm SD (n) & $P$ & mean $\pm S D(n)$ & $P$ & \\
\hline \multicolumn{6}{|l|}{ \% change HRSD } \\
\hline $0-I$ weeks $(n)$ & $31.8 \pm 21.3(22)$ & $<0.0001$ & $15.0 \pm 21.7(27)$ & 0.0003 & 0.0401 \\
\hline $0-2$ weeks (n) & $46.0 \pm 20.5(22)$ & $<0.0001$ & $27.5 \pm 31.6(27)$ & $<0.000$ I & 0.0181 \\
\hline $0-4$ weeks (n) & $54.5 \pm 29.5(22)$ & $<0.0001$ & $31.4 \pm 24.9(27)$ & $<0.0001$ & 0.0421 \\
\hline \multicolumn{6}{|l|}{$\%$ change MADRS } \\
\hline $0-I$ weeks (n) & $32.8 \pm 21.0(22)$ & $<0.0001$ & $18.1 \pm 24.7(27)$ & 0.0004 & 0.139 \\
\hline $0-2$ weeks (n) & $42.1 \pm 24.0(22)$ & $<0.0001$ & $28.9 \pm 30.0(27)$ & $<0.000$ I & 0.119 \\
\hline \multirow[t]{2}{*}{$0-4$ weeks (n) } & $52.6 \pm 26.8(22)$ & $<0.0001$ & $33.7 \pm 38.8(27)$ & $<0.0001$ & 0.171 \\
\hline & \multicolumn{2}{|c|}{ Mirtazapine $(n=22)$} & \multicolumn{2}{|c|}{ Duloxetine $(n=28)$} & $P$ \\
\hline Response rate (\%) & \multicolumn{2}{|l|}{59.1} & \multicolumn{2}{|l|}{28.6} & 0.127 \\
\hline Remission rate (\%) & \multicolumn{2}{|l|}{31.8} & \multicolumn{2}{|l|}{10.7} & 0.152 \\
\hline
\end{tabular}

Abbreviations: HRSD, Hamilton Rating Scale for Depression; MADRS, Montgomery-Åsberg Depression Rating Scale; MMRM, mixed-models repeated measures; SD, standard deviation.

in terms of between-group differences (Figure 3). Because three of the 17 items of the HRSD concern sleep disturbances and contribute to up to $11.5 \%$ of the total score, it has been hypothesized that the HRSD may favor sedating antidepressant drugs (eg, mirtazapine).

The percentage of remitters, particularly in the duloxetine subgroup, was surprisingly low compared with that in other studies. In the Phase III study of duloxetine, the change in the HRSD score in the annexation analysis was -10.2 after 6 weeks of administration. On the other hand, after 4 weeks of administration, we believe that it was lower $(-7.5)$ because the administration period was shorter. However, mirtazapine seems to be superior to duloxetine in terms of the response rate. A possible reason is that the antidepressant effect of mirtazapine may be faster than that of duloxetine (Figures 2 and 3).

Mirtazapine led to significantly greater somnolence than did duloxetine. The number of patients who took mirtazapine with sleeping pills was also marginally less than that in the duloxetine group. However, the reason for this was unclear. Likewise, a recent meta-analysis showed that mirtazapine caused more frequent sleepiness/ drowsiness/somnolence compared with SSRIs, but not compared with TCAs and SNRIs. ${ }^{2}$ Nausea occurred significantly more often with duloxetine than with mirtazapine. Another meta-analysis reported that nausea was the side effect most frequently associated with discontinuation of duloxetine treatment. ${ }^{3}$ Clinicians must pay particular attention to physical findings such as drowsiness and nausea in these patients.

Compared with other similar studies, ${ }^{2,3}$ both antidepressants were beneficial and relatively well tolerated in patients with MDD in Japan. Although it is well known that the prevalence of MDD in females is higher than that in males, the prevalence in males was higher than that in females in the present study. The reason for this is unclear. These results may be confounded by the following limitations. One of the main limitations of this study is the small sample size. Another limitation of the present study is that it did not include a placebo control arm and was not randomized to ensure that patients and prescribers were blinded to the medication assignment. Finally, sleeping pills were allowed during the study. Although we controlled for several confounding factors using multiple regression analysis, we cannot exclude confounding effects that sleeping pills may have had on the psychopathology ratings in those patients. Mirtazapine and duloxetine were beneficial and relatively well tolerated in patients with MDD. Given the small sample size and lack of a placebo, double-blind, randomized controlled clinical trials should be conducted to further explore the utility of mirtazapine and duloxetine in patients with MDD.

\section{Acknowledgments}

We thank Ms KitajimaT,Ms InoK, MsFukasawaT,MsOkudaM, Ms Matsudaira Y, Ms Nobata R, Ms Ieda H, Ms Tanooka S, Dr Mekata T, Ms Goto S, Ms Nishizawa E, Ms Ando Y, Dr Okumura T, Ms Matsumoto Y, Ms Tani M, Ms Isogai S, Ms Niwa M, Ms Kato Y, Mr Tanaka N, Ms Miyako M, Ms Shibata E, Ms Hayakawa N, and Ms Adachi A for their technical support.

\section{Disclosure}

Drs Kishi and Iwata declare that they have no direct conflict of interest or grant support that is directly related to the con- 
tent of the study. Dr Kishi has received speaker's honoraria from Astellas, Dainippon Sumitomo, Eli Lilly, Yoshitomi, Otsuka, Meiji, Shionogi, Novartis, and Pfizer. Dr Iwata has received speaker's honoraria from Astellas, Dainippon Sumitomo, Eli Lilly, GlaxoSmithKline, Janssen, Yoshitomi, Otsuka, Meiji, Shionogi, Novartis, and Pfizer. Drs Nagao, Funahashi, Hirano, and Fujita have nothing to disclose.

\section{Role of funding source}

None. This study was conducted within clinical practices. No pharmaceutical industry provided the drugs to the patients.

\section{References}

1. Croom KF, Perry CM, Plosker GL. Mirtazapine: a review of its use in major depression and other psychiatric disorders. CNS Drugs. 2009;23(5):427-452.
2. Watanabe N, Omori IM, Nakagawa A, et al. Mirtazapine versus other antidepressive agents for depression. Cochrane Database Syst Rev. 2011;12:CD006528.

3. Gartlehner G, Thaler K, Hansen RA, Gaynes BN. The general and comparative efficacy and safety of duloxetine in major depressive disorder: a systematic review and meta-analysis. Drug Saf. 2009;32(12): $1159-1173$

4. Cipriani A, Furukawa TA, Salanti G, et al. Comparative efficacy and acceptability of 12 new-generation antidepressants: a multiple-treatments meta-analysis. Lancet. 2009;373(9665):746-758.

5. Cookson J, Gilaberte I, Desaiah D, Kajdasz DK. Treatment benefits of duloxetine in major depressive disorder as assessed by number needed to treat. Int Clin Psychopharmacol. 2006;21(5):267-273.

6. Hamilton M. A rating scale for depression. J Neurol Neurosurg Psychiatry. 1960;23:56-62.

7. Montgomery SA, Asberg M. A new depression scale designed to be sensitive to change. Br J Psychiatry. 1979;134:382-389.

8. Kishi T, Kitajima T, Ikeda M, et al. CLOCK may predict the response to fluvoxamine treatment in Japanese major depressive disorder patients. Neuromolecular Med. 2009;11(2):53-57.

9. Thase ME, Entsuah AR, Rudolph RL. Remission rates during treatment with venlafaxine or selective serotonin reuptake inhibitors. $\mathrm{Br} J$ Psychiatry. 2001;178:234-241.

\section{Publish your work in this journal}

Neuropsychiatric Disease and Treatment is an international, peerreviewed journal of clinical therapeutics and pharmacology focusing on concise rapid reporting of clinical or pre-clinical studies on a range of neuropsychiatric and neurological disorders. This journal is indexed on PubMed Central, the 'PsycINFO' database and CAS.
The manuscript management system is completely online and includes a very quick and fair peer-review system, which is all easy to use. Visit http://www.dovepress.com/testimonials.php to read real quotes from published authors. 